\title{
Temporal and Spatial Dynamics of Peroxynitrite-Induced Oxidative Damage after Spinal Cord Contusion Injury
}

\author{
Kimberly M. Carrico, Radhika Vaishnav, and Edward D. Hall
}

\begin{abstract}
The reactive nitrogen species peroxynitrite $(\mathrm{PN})$ has been suggested to be an important mediator of the secondary oxidative damage that occurs following acute spinal cord injury (SCI). The PN decomposition products nitrogen dioxide $\left(\bullet \mathrm{NO}_{2}\right)$, hydroxyl radical $(\bullet \mathrm{OH})$, and carbonate radical $\left(\bullet \mathrm{CO}_{3}\right)$ are highly reactive with cellular lipids and proteins. In this immunohistochemical study, we examined the temporal $(3,24$, and $72 \mathrm{~h}$, and 1 and 2 weeks) and spatial relationships of $\mathrm{PN}$-mediated oxidative damage in the contusion-injured rat thoracic spinal cord (IH device, $200 \mathrm{kdyn}, \mathrm{T} 10)$ using 3-nitrotyrosine (3-NT), a marker for protein nitration by $\mathrm{PN}$-derived $\bullet \mathrm{NO}_{2}$ and 4hydroxynonenal (4-HNE), an indicator of lipid peroxidation (LP) initiated by any of the PN radicals. Minimal 3-NT or 4-HNE immunostaining was seen in sham, non-injured spinal cords. In contrast, both markers showed a substantial increase at $3 \mathrm{~h}$ post-injury at the epicenter, that extended throughout the gray matter and into the surrounding white matter. At 24 and $72 \mathrm{~h}$, the oxidative damage expanded circumferentially to involve all but a small rim of white matter tissue at the injury site, and longitudinally as much as 6-9 $\mathrm{mm}$ in the rostral and caudal directions. The staining was observed in neuronal soma, axons, and microvessels. At all time points except $3 \mathrm{~h}$, there was no significant difference in the mean rostral or caudal extent of 3-NT and 4-HNE staining. By 1, and more so at 2 weeks, the longitudinal extent of the oxidative damage staining was greatly decreased. The spatial and temporal overlap of 3-NT and 4-HNE staining supports the concept that PN is involved in both damage produced by lipid peroxidation and protein nitration, and that antioxidant agents that target $\mathrm{PN}$ or PN-derived radicals should be effective neuroprotectants for acute SCI if administered during the first post-injury hours.
\end{abstract}

Key words: 4-hydroxynonenal; lipid peroxidation; peroxynitrite; protein nitration; spinal cord injury; 3nitrotyrosine

\section{Introduction}

$\mathbf{T}$ HE ROLE OF REACTIVE OXYGEN-INDUCED oxidative damage to spinal cord lipids (i.e., lipid peroxidation, LP) and proteins following acute spinal cord injury (SCI) has been strongly supported in previous work (Aksenova et al., 2002; Baldwin et al., 1998; Braughler and Hall, 1989; Hall and Braughler, 1993; Juurlink and Paterson, 1998; Springer et al., 1997). Earlier investigations attributed much of the posttraumatic oxidative damage to the reactive oxygen species (ROS) hydrogen peroxide $\left(\mathrm{H}_{2} \mathrm{O}_{2}\right)$ and its derived hydroxyl radical $(\bullet \mathrm{OH})$ formed rapidly by iron-dependent Fenton reactions (Braughler and Hall, 1989). However, Beckman and coworkers (Beckman et al., 1990; Beckman, 1991) subsequently introduced the theory that another ROS that could logically be involved in producing secondary oxidative tissue injury was peroxynitrite (PN). Peroxynitrite, often referred to as a reactive nitrogen species, is formed by the diffusion ratelimited reaction of nitric oxide synthase (NOS)-generated nitric oxide $(\bullet \mathrm{NO})$ with superoxide radicals $\left(\mathrm{O}_{2}{ }^{--}\right)$at diffusionlimited rates to form $\mathrm{PN}$ anion $\left(\mathrm{ONOO}^{-}\right)$(Radi, 2004). However, the potent oxidizing ability of $\mathrm{PN}$ is actually due to its free radical decomposition products. These products are formed in two pathways. The first involves the protonation of $\mathrm{PN}$ to form peroxynitrous acid $(\mathrm{ONOOH})$, which can undergo homolytic decomposition to form the highly reactive nitrogen dioxide radical $\left(\bullet \mathrm{NO}_{2}\right)$ and hydroxyl radical $(\bullet \mathrm{OH})($ Beckman et al., 1990; Beckman, 2002; Radi et al., 2002b). In addition, PN will react with carbon dioxide $\left(\mathrm{CO}_{2}\right)$ to form nitrosoperoxocarbonate $\left(\mathrm{ONOOCO}_{2}{ }^{-}\right)$, which can then decompose into - $\mathrm{NO}_{2}$ and carbonate radical $\left(\mathrm{CO}_{3}{ }^{--}\right.$) (Deng et al., 2007; Radi, 2004; Squadrito and Pryor, 2002; Xiong et al., 2007). These radicals can initiate cellular damage by LP by attacking polyunsaturated fatty acids (Radi et al., 1991). The LP byproduct 4-hydroxynonenal (4-HNE), as well as other aldehydes formed from peroxidized lipids can then bind to cellular proteins compromising their structural and functional integrity (Halliwell and Gutteridge, 2007). Additionally, $\bullet \mathrm{NO}_{2}$

Spinal Cord \& Brain Injury Research Center (SCoBIRC), University of Kentucky College of Medicine, Lexington, Kentucky. 
can nitrate the 3 position of tyrosine residues in proteins (Radi et al., 2002a; Radi, 2004). Accordingly, the measurement of 3nitrotyrosine (3-NT) accumulation thus provides a biomarker of PN action (Beckman and Koppenol, 1996; Radi et al., 1991; Radi, 2004).

Prior studies in other laboratories (Liu et al., 2000; Scott et al., 1999) and our own (Xiong et al., 2007) have demonstrated 3-NT immunostaining in the injured spinal cord. Furthermore, recent work in our laboratory has provided convincing evidence for an important role of $\mathrm{PN}$ in the oxidative damage that occurs in the injured spinal cord and its relationship to other secondary injury events including intracellular calcium overload, mitochondrial dysfunction, and calpain-mediated cytoskeletal protein degradation (Xiong et al., 2007). The goal of the present experiments was to provide a more complete immunohistochemical definition of the time course, intensity and rostral-caudal extent of LP (4-HNE) and protein nitration (3-NT) in the contused rat spinal cord. The purpose was to more convincingly investigate the spatial and temporal overlap of the two oxidative injury mechanisms in order to support the hypothesis that PN is a major source of both in the injured spinal cord, and that PN-mediated damage affects both microvascular and parenchymal elements. The results would then enable the more rational design of PNtargeted antioxidant neuroprotective strategies.

\section{Methods}

\section{Subjects}

These experiments employed 24 young adult female Sprague-Dawley rats (Charles River, Portage, MI) weighing between 200 and $225 \mathrm{~g}$. The animals were randomly cycling and were not tested for stage of the estrus cycle. They were fed and watered ad libitum. All procedures described below have been approved by the University of Kentucky Institutional Animal Care and Use Committee, and followed NIH Guidelines for the Care and Use of Laboratory Animals.

\section{Rat contusion model of spinal cord injury}

The rats were anesthetized with ketamine $(80 \mathrm{mg} / \mathrm{kg}$ IP) and xylazine $(10 \mathrm{mg} / \mathrm{kg} \mathrm{IP})$. A laminectomy slightly larger than the $2.5-\mathrm{mm}$ impactor tip was made at the T10 vertebra and the vertebral column was stabilized by clamping the rostral T9 and caudal T11 vertebrae with forceps. The exposed cord was then manipulated to be level in the horizontal plane. A spinal cord contusion injury was then performed using the Infinite Horizons injury device (Scheff et al., 2003). This device applied a force-defined impact to the exposed spinal cord with a 2.5 -mm-diameter stainless steel-tipped impactor producing a reproducible contusion injury. The injury force used for this experiment was $200 \mathrm{kdyn}$, which produces a severe degree of contusion injury. A computer monitored the impact and recorded velocity and actual force of the impact, as well as the displacement of the spinal cord, allowing us to standardize the level of injury across all animals. Sham rats received the laminectomy but were not injured.

After injury, all rats received $20 \mathrm{~mL}$ of sterile lactated Ringer's solution injected SC bilaterally ( $10 \mathrm{~mL}$ per side) just rostral to the hind limbs to prevent dehydration, as well as $33.3 \mathrm{mg} / \mathrm{kg}$ of cefazolin also injected SC above the tail. They were then placed on $37^{\circ}$ water-circulating heating pads and returned to the animal room. The animals were housed two per cage until sacrifice, with postoperative animal care consisting of manual bladder expression, injection of $20 \mathrm{~mL}$ of lactated Ringer's solution, and injection of $33.3 \mathrm{mg} / \mathrm{kg}$ of cefazolin being performed twice daily until sacrifice or until 1 week post-injury. Any animal that showed signs of distress was also given $0.035 \mathrm{mg} / \mathrm{kg}$ of buprenorphine IM.

\section{Tissue processing}

At various time points after injury $(3,24$, and $72 \mathrm{~h}$, and 1 or 2 weeks) the rats ( $n=4 /$ time point) were deeply anesthetized with sodium pentobarbital ( $150 \mathrm{mg} / \mathrm{kg} \mathrm{IP})$ and transcardially perfused with $150 \mathrm{~mL}$ of $0.1 \mathrm{M}$ PBS followed by $200 \mathrm{~mL}$ of $4 \%$ paraformaldehyde. Four sham (laminectomized but noninjured) rats were also perfused at $24 \mathrm{~h}$ post-surgery. The spinal cord from the base of the skull to the cauda equina was then removed from the vertebral column and placed in $4 \%$ paraformaldehyde for $4 \mathrm{~h}$ before being transferred to $0.2 \mathrm{M}$ PBS for 1 to 5 days. Two days before being embedded in OTC (Tissue-Tek; Sakura Finetek USA, Inc., Torrance, CA), the cords were cryoprotected in $20 \%$ sucrose in $0.2 \mathrm{M}$ PBS. The tissue was cut into four sections as follows: (a) $3 \mathrm{~cm}$ centered on the injury epicenter, (b) $3 \mathrm{~cm}$ spanning 4.5 to $1.5 \mathrm{~cm}$ from the injury epicenter, (c) the remainder of the cord $4.5 \mathrm{~cm}$ rostral to the injury epicenter, and (d) the remainder of the cord $1.5 \mathrm{~cm}$ caudal to the injury epicenter. The excised cord sections were stored at $-80^{\circ} \mathrm{C}$ for several days. The spinal cords were sectioned in the transverse plane at $20 \mu \mathrm{m}$ thickness and every fifth section was placed directly onto a series of 10 Superfrost Plus Slides (Fisher Scientific, Hampton, NH). The slides were dehydrated overnight at $4^{\circ} \mathrm{C}$ before being stored at $-20^{\circ} \mathrm{C}$ until staining.

\section{Immunohistochemical analysis of 4-HNE and 3-NT}

The day of staining, three adjacent series of slides were thawed and stained for 4-HNE, 3-NT, and no-primary controls. Those being probed for 4-HNE were subjected to a reduction protocol using $0.1 \mathrm{M} \mathrm{NaBH}_{4}$ in $0.1 \mathrm{M} \mathrm{MOPS}$ (SigmaAldrich, St. Louis, MO). All slides were then incubated in $0.3 \%$ $\mathrm{H}_{2} \mathrm{O}_{2}$ in $0.2 \mathrm{M}$ PBS for $30 \mathrm{~min}$ and blocked in 5\% normal goat serum, $0.25 \%$ Triton $\mathrm{X}-100$, and $1 \%$ dry milk in $0.2 \mathrm{M}$ PBS for $2 \mathrm{~h}$ at room temperature. The slides were then incubated in the same blocking solution containing either anti-4hydroxynonenal (1:5000, rabbit anti-HNE Michael Adducts; EMD Chemicals, Gibbstown, NJ) or anti-nitrotyrosine (1:2000, rabbit anti-nitrotyrosine; Millipore Corp., Temecula, CA) antibodies overnight at $4{ }^{\circ} \mathrm{C}$. The no-primary controls were incubated in the blocking solution only. The next day, the tissue was placed in blocking solution containing biotinylated goat anti-rabbit secondary antibody (1 drop/20 mL, Vectastain $\mathrm{ABC}$-AP kit; Vector Laboratories, Burlingame, CA) for $2 \mathrm{~h}$ at room temperature before being incubated in Vectastain $\mathrm{ABC}$ reagent (1 drop $/ 5 \mathrm{~mL}$, Vectastain $\mathrm{ABC}$-AP kit; Vector Laboratories) for $1 \mathrm{~h}$. Staining was then visualized using the Vector Blue substrate kit (Vector Laboratories). Spinal cord sections were lightly counterstained with nuclear fast red (Vector Laboratories) and cover slipped with Permount.

Photomicrographs were taken using an Olympus Magnafire digital camera mounted on an Olympus Provis AX70 microscope (Olympus, Center Valley, PA) at either $4 \times, 20 \times$, or $40 \times$ magnification. To semi-quantitatively determine the 
anterior-posterior extent of the post-traumatic oxidative damage (4-HNE and 3-NT), all sections were examined for the presence of staining. A section was considered to indicate the presence of either marker if staining was present above the level found in the corresponding section from the sham, noninjured rats. Since only every fifth $20-\mu$ m section was stained, the rostral or caudal extent of the oxidative damage staining was determined by multiplying the number of successively stained sections by $100 \mu \mathrm{m}$. Accordingly, the rostral and caudal extents of 4-HNE and 3-NT staining were calculated for each animal ( $\mathrm{n}=4$ for each time-point), and the mean \pm standard error calculated for each post-traumatic time-point. Statistical comparison of the rostral and caudal extent of 4-HNE versus 3-NT immunostaining was carried out with paired $t$-tests at each time-point, with $p<0.05$ required for significance.

\section{Results}

\section{4-HNE and 3-NT immunostaining at the injury epicenter}

This study showed that both 4-HNE and 3-NT accumulate rapidly after spinal cord injury, increasing as early as $3 \mathrm{~h}$ postinjury, with 3-NT remaining elevated until 1 week post-injury, and 4-HNE persisting until at least 2 weeks post-injury. The upper panels of Figure 1 show a comparison of representative coronal sections taken at the injury epicenter (T10) at 3, 24, and $72 \mathrm{~h}$, and 1 and 2 weeks post-injury and stained for 4-HNE. The 4-HNE immunoreactivity was minimal in sham tissue harvested $24 \mathrm{~h}$ post-surgery. However, as early as $3 \mathrm{~h}$ postinjury, 4-HNE immunoreactivity was increased, with intense staining encompassing all of the gray matter and extending into the surrounding white matter. Staining peaked at 24 to $72 \mathrm{~h}$ post-injury, at which time there was only a small rim of white matter remaining unstained. The 4-HNE staining persisted out to 2 weeks post-injury, with a large portion of the gray matter still showing staining. The 3-NT immunoreactivity (lower panels of Fig. 1) showed a similar pattern to that of 4-HNE, with little to no reactivity in sham tissue. As with the $4-\mathrm{HNE}$, there was an increase in staining as early as $3 \mathrm{~h}$ post-injury, which peaked between 24 and $72 \mathrm{~h}$ post-injury. By 1 week post-injury, however, 3-NT staining had nearly disappeared, with only a small amount of gray matter showing staining, and by 2 weeks post-injury, immunoreactivity had returned to sham levels.

\section{Rostral and caudal extent of 4-HNE and 3-NT immunostaining}

The rostral-caudal extent of 4-HNE immunoreactivity is shown in Figure 2. Again, the sham animals show minimal background staining confined mostly to the gray matter. While staining was most intense at the injury epicenter (T10) at every time point, images taken every $2 \mathrm{~mm}$ show that immunoreactivity at 3,24 , and $72 \mathrm{~h}$ post-injury extends at least $6 \mathrm{~mm}$ in both the rostral and caudal directions. At 1 week post-injury, the epicenter still shows strong reactivity. However, staining in the rostral direction was reduced slightly when compared to the 72 -h sections, and staining in the caudal direction had returned to sham levels. By 2 weeks postinjury, persistent reactivity was localized to the epicenter and sections immediately adjacent.

The 3-NT immunoreactivity showed a similar pattern to that of 4-HNE (Fig. 3). Reactivity in sham tissue was absent, and again the most intense staining at every time-point was found at the T10 epicenter. At $3 \mathrm{~h}$ post-injury, staining could be found in sections as much as $2-3 \mathrm{~mm}$ rostral to the epicenter and $6 \mathrm{~mm}$ caudal to the epicenter. By $24 \mathrm{~h}$ post-injury, staining in most animals extended $6 \mathrm{~mm}$ in either direction, a pattern that persisted to $72 \mathrm{~h}$ post-injury. In contrast to the 3NT immunoreactivity, however, by 1 week post-injury staining had mostly disappeared and was confined to sections within $2 \mathrm{~mm}$ of the epicenter. Tissue harvested 2 weeks postinjury showed little to no reactivity in any sections.

The graphs in Figure 4 show a quantitative representation of the rostral-caudal distribution of both 4-HNE (top panel) and 3-NT (bottom panel) across the examined post-injury time-points ( $\mathrm{n}=4$ for each). Sham animals showed no immunoreactivity and so were not included in the graphs. At $3 \mathrm{~h}$


Time course of 4-HNE and 3-NT at the epicenter

FIG. 1. Representative examples showing the post-traumatic time course of oxidative damage as revealed by 3-NT and 4HNE immunostaining. Images are representative coronal sections at the epicenter (T10) at 3, 24, and $72 \mathrm{~h}$ and at 1 and 2 weeks post-injury. Adjacent sections from the same animal were stained for 3-NT and 4-HNE. All sections were counterstained with nuclear fast red. The sham animal showed minimal staining for both markers. By $3 \mathrm{~h}$ post-injury, staining was substantially elevated, encompassing all of the gray matter and extending into the white matter. It peaked for 3-NT and 4-HNE around 24 to $72 \mathrm{~h}$ post-injury, with only a small rim of white matter remaining unstained. By 1 week, 3-NT staining had nearly disappeared, while the elevation of 4-HNE persisted throughout the gray matter until at least 2 weeks post-injury (scale bar $=500 \mu \mathrm{m})$. 




Rostral-caudal expanse of 4-HNE staining



$-2 \mathrm{~mm}$

$-4 \mathrm{~mm}$

$-6 \mathrm{~mm}$

post-injury, 4-HNE reactivity was found up to $6 \mathrm{~mm}$ in the rostral and caudal directions. At $24 \mathrm{~h}$ and $72 \mathrm{~h}$ post-injury, the rostral distribution was much the same $(6 \mathrm{~mm})$, but tissue as much as $9 \mathrm{~mm}$ in the caudal direction showed reactivity. By 1 week post-injury, staining was confined to sections $4 \mathrm{~mm}$ from the epicenter rostrally, and only $2 \mathrm{~mm}$ from the epicenter caudally. At 2 weeks post-injury, the area where reactivity occurred was further reduced, as staining was limited to an area within $1 \mathrm{~mm}$ of the epicenter in either direction. The mean rostral and caudal extents of 3-NT immunoreactivity were not significantly different than that of 4 -HNE except at the initial (3-h) time-point when the rostral spread of 3-NT staining only extended $3 \mathrm{~mm}$ from the epicenter, compared to $6 \mathrm{~mm}$ for 4 -HNE $(p<0.03)$. However, no significant difference was seen in the caudal distribution of 3-NT and 4-HNE at the 3-h time-point. Moreover, no differences in mean rostral or caudal extents of 3-NT versus 4-HNE were seen at any of the later time-points. At $24 \mathrm{~h}$ post-injury, 3-NT reactivity was found $4 \mathrm{~mm}$ rostral and $12 \mathrm{~mm}$ caudal to the epicenter. By $72 \mathrm{~h}$ post-injury the rostral-caudal extent of staining had decreased, and by 1 week post-injury, staining extended only $2 \mathrm{~mm}$ in either direction. At 2 weeks post-injury, only a small amount of staining was seen, localized to the injury epicenter.

\section{Microvascular and parenchymal 3-NT immunostaining}

As shown in Figure 5, higher-power (20x and 40x) images from either 24 or $72 \mathrm{~h}$ post-injury were also examined for the purpose of identifying the relative 3-NT staining of microvascular versus parenchymal elements. The top row shows a 24-h post-injury spinal cord cross-section at $1 \mathrm{~mm}$ caudal to the epicenter. The $4 \times$ photo shows intense 3 -NT staining of ventral horn motor neuron cell bodies. The $20 \times$ and $40 \times$ photos show three heavily stained microvessels (arrows). The middle row displays three magnifications of a 72-h post-injury crosssection $1 \mathrm{~mm}$ rostral to the epicenter, which shows an intensely 3-NT-stained microvessel (arrow). The 40× inset more clearly shows that the 3-NT staining in the microvessel wall extends out into the parenchyma, with gradually decreasing intensity as the distance from the vessel increases. This suggests that the microvessels may be a primary site of oxidative damage. The bottom row shows examples at $24 \mathrm{~h}$ that were taken $3 \mathrm{~mm}$ caudal to the epicenter, in which intense 3-NT staining of presumptive dorsal commissural axons and cell bodies is apparent. These examples illustrate that both microvessels and neuronal elements are targets of nitrative damage.

\section{Discussion}

Previous studies have shown that there is an increase in 3NT levels in spinal cord tissue during the first hours and days after acute contusion SCI (Bao et al., 2003; Liu et al., 2000; Scott et al., 1999; Scott et al., 2003; Xiong et al., 2007). Additional work has demonstrated that the infusion of PN at levels that mimic the estimated concentration produced in the contused spinal cord produces nitrative immunostaining and neuronal damage similar to that produced by contusion injury (Liu et al., 2005). The increase in 3-NT is paralleled by increases in LP products (Xiong et al., 2007) and the activation of the DNA repair enzyme poly-ADP ribose polymerase (Scott et al., 1999; Scott et al., 2004); both of these pathochemical events are known to be capable of being produced by PN (Beckman et al., 1990; Beckman, 1991; Beckman, 2002; Pacher et al., 2007; Radi et al., 1991). A recent report from our laboratory has shown that the coincident increase in the levels of 3-NT and the LP product 4-HNE during the first hour after injury persists at least until 7 days post-injury (Xiong et al., 2007). However, none of these prior reports has fully documented the spatial and temporal characteristics of PN-mediated oxidative damage in the injured cord related to protein nitration (3-NT) and LP (4-HNE).

Filling this knowledge gap, the current results show that contusion SCI increased 4-HNE and 3-NT substantially above sham levels (with minimal staining) as early as $3 \mathrm{~h}$ post-injury that peaked at 24 and $72 \mathrm{~h}$ post-injury. An increased level of staining persisted up to 1 week for 3-NT and for at least 2 weeks for 4-HNE. Oxidative damage staining was initially most intense in the gray matter and inner-most portions of white matter, but spread outwardly in white matter over time. The 4-HNE staining extended from $6 \mathrm{~mm}$ rostral to $9 \mathrm{~mm}$ caudal to the epicenter, with the largest extension found at 24 and $72 \mathrm{~h}$ post-injury. 3-NT showed a similar distribution, with the peak distribution occurring at 24 and $72 \mathrm{~h}$ and appearing as much as $6 \mathrm{~mm}$ rostral to the epicenter and $12 \mathrm{~mm}$ in the caudal direction. The overlap of $4-\mathrm{HNE}$ and 3-NT staining temporally as well as spatially suggests that $\mathrm{PN}$ contributes to LP as well as protein nitration after SCI. Importantly, the 3-NT and 4-HNE immunohistochemistry demonstrates that PNmediated oxidative damage simultaneously affects spinal cord microvessels and neuronal soma, dendrites, and axons. This broad spectrum of PN cellular damage has also been seen in the injured brain (Deng et al., 2007; Hall et al., 2004). This suggests that therapeutic attempts to inhibit oxidative damage by PN need to target both the microvasculature and the neural parenchyma.

Recent experiments have strongly indicated that a key cellular source of post-traumatic PN in either spinal cord or brain injury are mitochondria. Mitochondria have been demonstrated to contain a constitutive NOS isoform that is activated by traumatically-induced intracellular calcium, and that the resulting increase in $\bullet \mathrm{NO}$ together with an increase in mitochondrial leakage of $\mathrm{O}_{2}{ }^{\bullet-}$ leads to PN generation (Deng

FIG. 2. Rostral-caudal extent of 4-HNE staining at all time-points as shown by immunohistochemistry. All sections were counterstained with nuclear fast red. Images were taken every $2 \mathrm{~mm}$ starting $6 \mathrm{~mm}$ rostral to the epicenter and ending $6 \mathrm{~mm}$ caudal to the epicenter. At each time point, the images were taken from only one animal. By $3 \mathrm{~h}$ post-injury, staining was elevated above sham levels at the epicenter, with this elevation present at least $6 \mathrm{~mm}$ in both the rostral and caudal directions. This staining pattern persisted up to $72 \mathrm{~h}$ post-injury, with 4-HNE indicated at least as much as $6 \mathrm{~mm}$ from the epicenter in both directions. At 1 week post-injury, staining was still intense $2 \mathrm{~mm}$ rostral to the epicenter, but had abated somewhat by $4 \mathrm{~mm}$, although it remained above sham levels out to $6 \mathrm{~mm}$ rostrally. Very little staining was seen at 1 week post-injury in the caudal direction. By 2 weeks post-injury, staining was still present at the epicenter, but was not seen more than $2 \mathrm{~mm}$ in either direction. Compare Figure 2 with Figure 3, which shows protein nitration-related 3-NT staining of the adjacent sections (scale bar $=500 \mu \mathrm{m})$. 
Rostral-caudal extent of 3-NT staining



\section{Epicenter Sham}

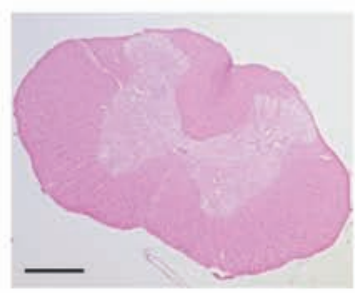

$3 \mathbf{h}$


$24 \mathrm{~h}$



$72 \mathrm{~h}$



1 wk



2 wk



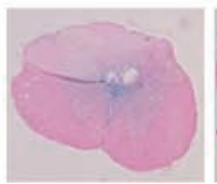
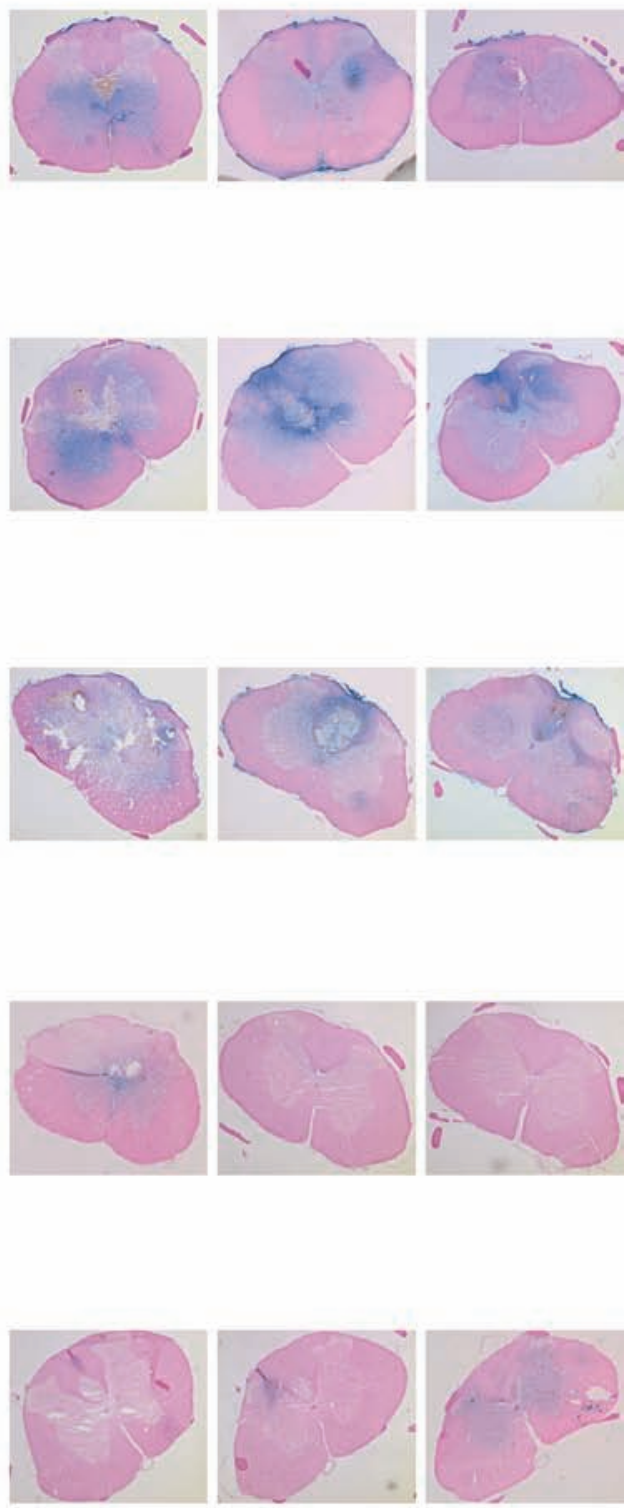

Caudal

FIG. 3. Rostral-caudal extent of 3-NT staining at all time-points as shown by immunohistochemistry. All sections were counterstained with nuclear fast red. Images were taken every $2 \mathrm{~mm}$ starting $6 \mathrm{~mm}$ rostral to the epicenter, and ending $6 \mathrm{~mm}$ caudal to the epicenter. At each time-point, the images were taken from only one animal. By $3 \mathrm{~h}$ post-injury, staining was elevated above sham levels at the epicenter and for several millimeters in both the rostral and caudal directions. The largest spread of staining was present between 24 and $72 \mathrm{~h}$ post-injury, with 3-NT apparent at least $6 \mathrm{~mm}$ from the epicenter in either direction. By 1 week post-injury, staining had largely abated and was found sparingly and only in sections within $2 \mathrm{~mm}$ of the epicenter. At 2 weeks post-injury staining had returned to near-sham levels even at the epicenter. (Compare Figure 3 with Figure 2, which shows lipid peroxidative-related 4-HNE staining of the adjacent sections [scale bar $=500 \mu \mathrm{m}$ ]). 


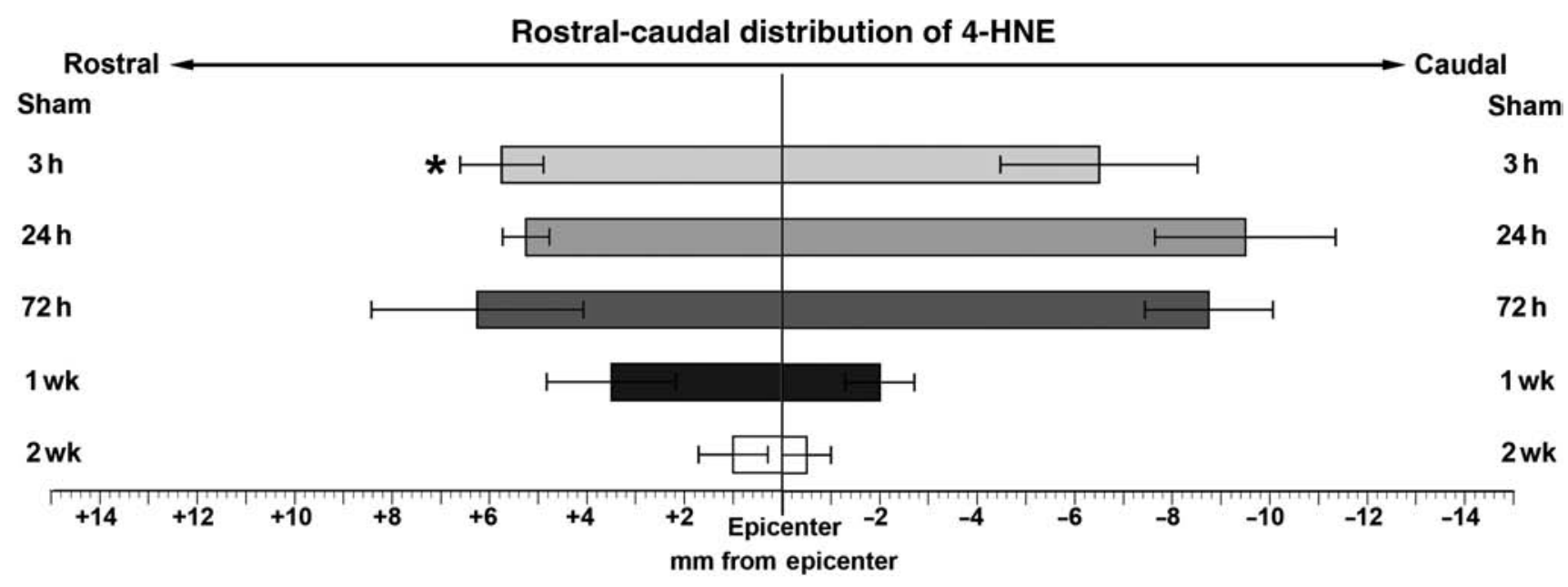

Rostral-caudal distribution of 3-NT



FIG. 4. Graphic summary of the rostral-caudal distribution of staining for both 4-HNE and 3-NT over various time-points in the injured rat spinal cord (values $=$ mean \pm SEM for 4 animals/time-point). The extent of staining for 4-HNE and 3-NT are expressed in millimeters from the epicenter $(0)$ in both the rostral $(+)$ and caudal (-) directions. See methods section for details on measurements. The top panel shows the extent of 4-HNE staining, with the peak of its distribution between 24 and $72 \mathrm{~h}$ post-injury and some staining found up to $1 \mathrm{~mm}$ from the epicenter as late as 2 weeks post-injury. The bottom panel shows the extent of 3-NT staining, with the peak again occurring between 24 and $72 \mathrm{~h}$ post-injury. At 1 week post-injury, some staining was present up to $2 \mathrm{~mm}$ from the epicenter, but by 2 weeks post-injury, staining was found only in the epicenter sections $\left({ }^{*} p<0.05\right.$ versus 4 -HNE at the same time-point by paired $t$-test).

et al., 2007; Hall et al., 2004; Radi et al., 2002b; Xiong et al., 2007). Consistent with this scenario, SCI and TBI initiate mitochondrial respiratory and calcium-buffering dysfunction during the first few post-traumatic hours, together with an increase in 3-NT and 4-HNE modification of mitochondrial proteins (Deng et al., 2007; Singh et al., 2006; Xiong et al., 2007). Similarly, exposure of normal (non-injured) CNS mitochondria to $\mathrm{PN}$ replicates post-traumatic mitochondrial respiratory dysfunction and 3-NT modification of mitochondrial proteins (Singh et al., 2007). Thus, mitochondria appear to be both a source of post-traumatic PN generation, and are likely an initial site of PN-mediated oxidative damage. This leads to loss of normal oxidative phosphorylation (i.e., ATP synthesis) and calcium-buffering capacity that becomes apparent as early as $6 \mathrm{~h}$ and peaks at $24 \mathrm{~h}$ post-injury (Sullivan et al., 2007). However, while the mitochondrial generation of PN may be important during the first $24 \mathrm{~h}$ after SCI, there is considerable evidence that the generation of $\mathrm{PN}$ is also contributed to by delayed cellular inflammatory mechanisms involving activation of inducible nitric oxide synthase (iNOS). For example, iNOS enzymatic activity has been shown to be increased in the contused rat spinal cord as early as $24 \mathrm{~h}$ post-injury, coincident with the invasion of polymorphonuclear leukocytes (PMNs), and administration of the selective iNOS inhibitor aminoguanidine can improve neurological and histological outcomes (Chatzipanteli et al., 2002). A second group has demonstrated upregulation of iNOS expression as early as $2 \mathrm{~h}$ after spinal cord contusion injury that peaks at $72 \mathrm{~h}$. A third set of investigators has reported that the increase in 3-NT until 7 days occurs together with an increase in the levels of iNOS in activated microglia in the injured cord (Xu et al., 2001). Thus it would appear that 


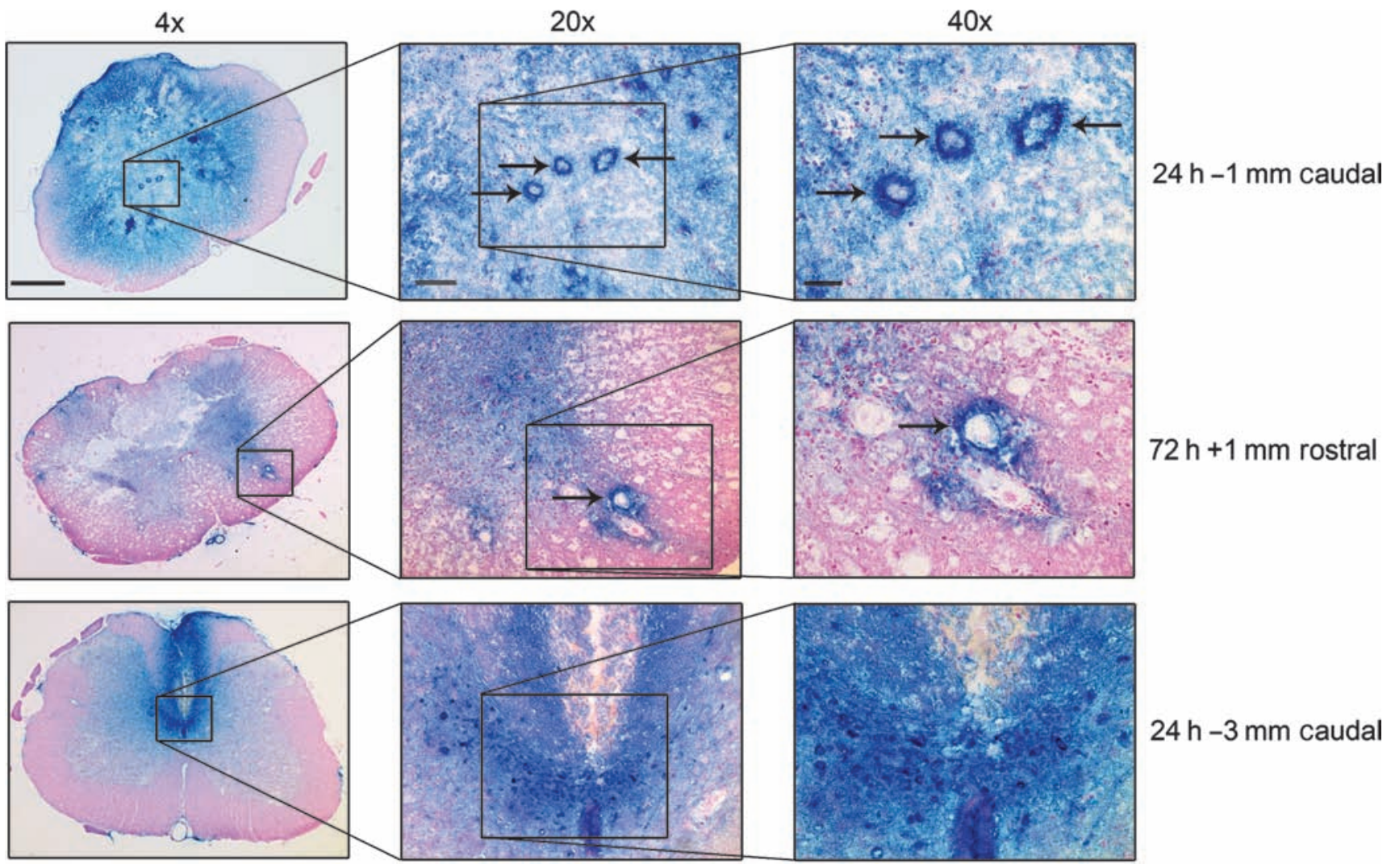

FIG. 5. Higher-power views of 3-NT oxidative damage in the injured spinal cord. The top row shows a 24-h post-injury spinal cross-section at $1 \mathrm{~mm}$ caudal to the epicenter, and shows intense oxidative damage staining of gray matter microvessels (arrows), as well as surrounding parenchymal elements. The middle row displays a 72-h post-injury cross-section $1 \mathrm{~mm}$ rostral to the epicenter, which again shows an example of microvascular staining (arrow). The bottom row shows an example at $24 \mathrm{~h}$ that was taken $3 \mathrm{~mm}$ caudal to the epicenter, in which intense 3-NT staining of presumptive dorsal commissural axons and cell bodies is apparent (4× scale bar $=500 \mu \mathrm{m} ; 20 \times$ scale bar $=200 \mu \mathrm{m} ; 40 \times$ scale bar $=100 \mu \mathrm{m}$ ).

over much of the acute (first $24 \mathrm{~h}$ ) and subacute $(24 \mathrm{~h}$ to 1 week) SCI time course, the generation of $\bullet \mathrm{NO}$, and ultimately $\mathrm{PN}$, comes at least from a combination of mitochondrial constitutive NOS activation and inflammatory cell iNOS upregulation (Conti et al., 2007). Furthermore, keep in mind that some of the $\cdot \mathrm{NO}_{2}$ that mediates tyrosine nitration can be generated from the heme-containing myeloperoxidase (MPO) in either infiltrating PMNs or macrophages, as well as in activated spinal cord microglia. The chemistry of this enzymatic source involves MPO-catalyzed conversion of nitrite anion $\left(\mathrm{NO}_{2}{ }^{-}\right)$to $\bullet \mathrm{NO}_{2}$ (Radi, 2004). This non-PN source of 3-NT is more likely to contribute at $24 \mathrm{~h}$ or later post-injury, the times when the most inflammatory cell infiltration occurs (Donnelly and Popovich, 2008).

The current results, as well as those of earlier studies (Bao et al., 2003; Genovese et al., 2007; Liu et al., 2005; Scott et al., 1999; Scott et al., 2003; Scott et al., 2005; Xiong et al., 2007; Xiong and Hall, 2009), suggest that the administration of antioxidants that target $\mathrm{PN}$ or PN-derived radicals within the first hours after SCI should provide effective neuroprotection. In agreement with this idea, compounds that can either scavenge peroxynitrite such as uric acid (Scott et al., 2005) or FeTSPP (Genovese et al., 2007), or its derived free radicals such as tempol (Hillard et al., 2004; Xiong and Hall, 2009) have been reported to attenuate post-SCI spinal tissue damage. The neuroprotective effects of the latter compound, tempol, have been recently shown to occur together with a decrease in mitochondrial dysfunction, protein oxidative damage, and calcium-mediated cytoskeletal proteolytic degradation (Xiong et al., 2007; Xiong and Hall, 2009). In any event, the current results strongly suggest that future therapeutic attempts to inhibit post-traumatic oxidative damage in SCI need to (1) be directed at PN or its derived free radicals; (2) be initiated as early as possible, ideally within the first few hours; (3) protect both microvascular and neuronal cells; and (4) may need to be continued for as long as 7-14 days post-injury.

\section{Acknowledgments}

This work was supported by a grant from the Kentucky Spinal Cord \& Head Injury Research Trust (5P30 NS051220).

\section{Author Disclosure Statement}

No conflicting financial interest exist.

\section{References}

Aksenova, M., Butterfield, D.A., Zhang, S.X., Underwood, M., and Geddes, J.W. (2002). Increased protein oxidation and decreased creatine kinase BB expression and activity after spinal cord contusion injury. J. Neurotrauma 19, 491-502. 
Baldwin, S.A., Broderick, R., Osbourne, D., Waeg, G., Blades, D.A., and Scheff, S.W. (1998). The presence of 4-hydroxynonenal/ protein complex as an indicator of oxidative stress after experimental spinal cord contusion in a rat model. J. Neurosurg. 88, 874-883.

Bao, F., DeWitt, D.S., Prough, D.S., and Liu, D. (2003). Peroxynitrite generated in the rat spinal cord induces oxidation and nitration of proteins: reduction by Mn (III) tetrakis (4-benzoic acid) porphyrin. J. Neurosci. Res. 71, 220-227.

Beckman, J.S., Beckman, T.W., Chen, J., Marshall, P.A., and Freeman, B.A. (1990). Apparent hydroxyl radical production by peroxynitrite: implications for endothelial injury from nitric oxide and superoxide. Proc. Natl. Acad. Sci. U.S.A. 87, 16201624.

Beckman, J.S., and Koppenol, W.H. (1996). Nitric oxide, superoxide, and peroxynitrite: the good, the bad, and ugly. Am. J. Physiol. 271, C1424-C1427.

Beckman, J.S. (2002). Protein tyrosine nitration and peroxynitrite. FASEB J. 16, 1144.

Beckman, J.S. (1991). The double-edged role of nitric oxide in brain function and superoxide-mediated injury. J. Dev. Physiol. 15, 53-59.

Braughler, J.M., and Hall, E.D. (1989). Central nervous system trauma and stroke. I. Biochemical considerations for oxygen radical formation and lipid peroxidation. Free Radic. Biol. Med. 6, 289-301.

Chatzipanteli, K., Garcia, R., Marcillo, A.E., Loor, K.E., Kraydieh, S., and Dietrich, W.D. (2002). Temporal and segmental distribution of constitutive and inducible nitric oxide synthases after traumatic spinal cord injury: effect of aminoguanidine treatment. J. Neurotrauma 19, 639-651.

Conti, A., Miscusi, M., Cardali, S., Germanò, A., Suzuki, H., Cuzzocrea, S., and Tomasello, F. (2007). Nitric oxide in the injured spinal cord: synthases cross-talk, oxidative stress and inflammation. Brain Res. Rev. 54, 205-218.

Deng, Y., Thompson, B.M., Gao, X., and Hall, E.D. (2007). Temporal relationship of peroxynitrite-induced oxidative damage, calpain-mediated cytoskeletal degradation and neurodegeneration after traumatic brain injury. Exp. Neurol. 205, 154-165.

Donnelly, D.J., and Popovich, P.G. (2008). Inflammation and its role in neuroprotection, axonal regeneration and functional recovery after spinal cord injury. Exp. Neurol. 209, 378-838.

Genovese, T., Mazzon, E., Esposito, E,. Muia, C., Di Paola, R., Bramanti, P., and Cuzzocrea, S. (2007). Beneficial effects of FeTSPP, a peroxynitrite decomposition catalyst, in a mouse model of spinal cord injury. Free Rad. Bio. Med. 43, 763-780.

Hall, E.D., and Braughler, J.M. (1993). Free radicals in CNS injury. Res. Publ. Assoc. Res. Nerv. Ment. Dis. 71, 81-105.

Hall, E.D., Detloff, M.R., Johnson, K., and Kupina, N.C. (2004). Peroxynitrite-mediated protein nitration and lipid peroxidation in a mouse model of traumatic brain injury. J. Neurotrauma 21, 9-20.

Halliwell, B., and Gutteridge, J.M.C. (2007). Free Radicals in Biology and Medicine, 4th ed. Oxford: Oxford University Press. Hillard, V.H., Peng, H., Zhang, Y., Das, K., Murali, R., Etlinger, J.D., and Zeman, R.J. (2004). Tempol, a nitroxide antioxidant, improves locomotor and histological outcomes after spinal cord contusion in rats. J. Neurotrauma 21, 1405-1414.

Juurlink, B.H., and Paterson, P.G. (1998). Review of oxidative stress in brain and spinal cord injury: suggestions for pharmacological and nutritional management strategies. J. Spinal Cord Med. 21, 309-334.
Liu, D., Ling, X., Wen, J., and Liu, J. (2000). The role of reactive nitrogen species in secondary spinal cord injury: formation of nitric oxide, peroxynitrite, and nitrated protein. J. Neurochem. 75, 2144-2154.

Liu, D., Bao, F., Prough, D.S., and Dewitt, D.S. (2005). Peroxynitrite generated at the level produced by spinal cord injury induces peroxidation of membrane phospholipids in normal rat cord: reduction by a metalloporphyrin. J. Neurotrauma 22, 1123-1133.

Pacher, P., Beckman, J.S., and Liaudet, L. (2007). Nitric oxide and peroxynitrite in health and disease. Physiol. Rev. 87, 315-424.

Radi, R., Beckman, J.S., Bush, K.M., and Freeman, B.A. (1991). Peroxynitrite-induced membrane lipid peroxidation: the cytotoxic potential of superoxide and nitric oxide. Arch. Biochem. Biophys. 288, 481-487.

Radi, R., Cassina, A., and Hodara, R. (2002a). Nitric oxide and peroxynitrite interactions with mitochondria. Biol. Chem. 383, 401-409.

Radi, R., Cassina, A., Hodara, R., Quijano, C., and Castro, L. (2002b). Peroxynitrite reactions and formation in mitochondria. Free Rad. Biol. Med. 33, 1451-1464.

Radi, R. (2004). Nitric oxide, oxidants, and protein tyrosine nitration. Proc. Natl. Acad. Sci. U.S.A. 101, 4003-4008.

Scheff, S.W., Rabchevsky, A.G., Fugaccia, I., Main, J.A., and Lumpp, J.E. (2003). Experimental modeling of spinal cord injury: characterization of a force-defined injury device. J. Neurotrauma 20, 179-193.

Scott, G.S., Jakeman, L.B., Stokes, B.T., and Szabo, C. (1999). Peroxynitrite production and activation of poly (adenosine diphosphate-ribose) synthetase in spinal cord injury. Ann. Neurol. 45, 120-124.

Scott, G.S., Virag, L., Szabo, C., and Hooper, D.C. (2003). Peroxynitrite-induced oligodendrocyte toxicity is not dependent on poly(ADP-ribose) polymerase activation. Glia 41, 105-116.

Scott, G.S., Szabo, C., and Hooper, D.C. (2004). Poly(ADPribose) polymerase activity contributes to peroxynitriteinduced spinal cord neuronal cell death in vitro. J. Neurotrauma 21, 1255-1263.

Scott, G.S., Cuzzocrea, S., Genovese, T., Koprowski, H., and Hooper, D.C. (2005). Uric acid protects against secondary damage after spinal cord injury. Proc. Natl. Acad. Sci. U.S.A. $102,3483-3488$

Singh, I.N., Sullivan, P.G., Deng, Y., Mbye, L.H., and Hall, E.D. (2006). Time course of post-traumatic mitochondrial oxidative damage and dysfunction in a mouse model of focal traumatic brain injury: implications for neuroprotective therapy. J. Cereb. Blood Flow Metab. 26, 1407-1418.

Singh, I.N., Sullivan, P.G., and Hall, E.D. (2007). Peroxynitritemediated oxidative damage to brain mitochondria: Protective effects of peroxynitrite scavengers. J. Neurosci. Res. 85, 22162223.

Springer, J.E., Azbill, R.D., Mark, R.J., Begley, J.G., Waeg, G., and Mattson, M.P. (1997). 4-Hydroxynonenal, a lipid peroxidation product, rapidly accumulates following traumatic spinal cord injury and inhibits glutamate uptake. J. Neurochem. 68, 24692476.

Squadrito, G.L., and Pryor, W.A. (2002). Mapping the reaction of peroxynitrite with $\mathrm{CO}_{2}$ : energetics, reactive species, and biological implications. Chem. Res. Toxicol. 15, 885-895.

Sullivan, P.G., Krishnamurthy, S., Patel, S.P., Pandya, J.D., and Rabchevsky, A.G. (2007). Temporal characterization of mitochondrial bioenergetics after spinal cord injury. J. Neurotrauma 24, 991-999. 
Xiong, Y., Rabchevsky, A.G., and Hall, E.D. (2007). Role of peroxynitrite in secondary oxidative damage after spinal cord injury. J. Neurochem. 100, 639-649.

Xiong, Y., and Hall, E.D. (2009). Pharmacological evidence for a role of peroxynitrite in the pathophysiology of spinal cord injury. Exp. Neurol. 216, 105-114.

Xu, J., Gyeong-Moon, K., Chen, S., Yan, P., Hinan, A., Ku, G., Beckman, J.S., Xu, X., and Hsu, C. (2001). iNOS and nitrotyrosine expression after spinal cord injury. J. Neurotrauma 18, 523-532.
Address correspondence to: Edward D. Hall, Ph.D. Director and Professor Spinal Cord E Brain Injury Research Center 741 S. Limestone Street BBSRB Room 483 Lexington, KY 40536-0509

E-mail: edhall@uky.edu 